\title{
Molecular Observation of Contour-Length Fluctuations Limiting Topological Confinement in Polymer Melts
}

\author{
A. Wischnewski, M. Monkenbusch, L. Willner, and D. Richter \\ Institut für Festkörperforschung, Forschungszentrum Jülich, D-52425 Jülich, Germany \\ A.E. Likhtman and T.C. B. McLeish \\ IRC in Polymer Science and Technology, University of Leeds, Leeds LS2 9JT, United Kingdom \\ B. Farago \\ Institut Laue-Langevin, B.P. 156X, Avenue des Martyrs, F-38042 Grenoble Cedex 9, France
}

(Received 19 October 2001; published 17 January 2002)

\begin{abstract}
In order to study the mechanisms limiting the topological chain confinement in polymer melts, we have performed neutron-spin-echo investigations of the single-chain dynamic-structure factor from polyethylene melts over a large range of chain lengths. While at high molecular weight the reptation model is corroborated, a systematic loosening of the confinement with decreasing chain length is found. The dynamic-structure factors are quantitatively described by the effect of contour-length fluctuations on the confining tube, establishing this mechanism on a molecular level in space and time.
\end{abstract}

DOI: $10.1103 /$ PhysRevLett.88.058301

PACS numbers: $83.80 . \mathrm{Sg}, 61.12 .-\mathrm{q}$

In the melt, long chain polymers heavily interpenetrate each other and mutually restrict their motions in forming topological constraints. In his famous reptation model, de Gennes described the effect of these entanglements by a tube along the coarse grained chain profile localizing the chain and confining the chain motion [1,2]. The dominating motional mechanisms in this model are (i) a curvilinear version of the Rouse motion at short times (also termed local reptation), and (ii) escape of the whole molecule from the tube at long times - the reptation process. Although the reptation model treated at this level is able to describe macroscopic melt dynamics qualitatively, as expressed by measurements of rheology and diffusion, it was shown by Doi a long time ago [3] that, in order to achieve even semiquantitative agreement with the rheology of polymer melts at time scales less than Rouse time, one needs an additional mechanism of chain motion, namely, contour-length fluctuations. Rheology is an indirect probe of molecular motion, but very recently it became possible to observe the tube confinement and the associated restrictions of segment motion directly in space and time [4-6].

Exploiting the new capabilities of ultrahigh resolution neutron-spin-echo (NSE) spectroscopy the dynamicstructure factor of polyethylene (PE) was explored deeply in the entangled regime. Schleger et al. showed that the dynamic-structure factor of the reptation model was corroborated, while all other competing models, which predict structure factors, performed far less well [6].

Thus, the assumption of tube confinement, enforcing one-dimensional (1D) chain motion along the tube, is very effective in describing the chain dynamics for long chains. However, as is known from broad cross-over phenomena such as the molecular weight dependence of the melt viscosity, very important limiting mechanisms exist which affect the confinement and limit the reptation process [2].
These processes increase in importance as the chain length decreases. Theoretically, constraint release, where a constraining chain moves out of the way of a given chain, and tube contour-length fluctuations removing the constraints from the ends have been identified as the most important of those processes but no direct microscopic observation exists.

In this paper we address the contour-length fluctuation mechanism. We present a systematic study of the singlechain dynamic-structure factor of PE melts for weight averaged molecular weights between $12.4 \mathrm{~kg} / \mathrm{mol} \leq M_{w} \leq$ $190 \mathrm{~kg} / \mathrm{mol}$ with a narrow distribution $M_{w} / M_{n}<1.05$. While at high $M_{w}$ only "local reptation" is active and the de Gennes model is confirmed; with decreasing $M_{w}$ we observe a gradual speedup of the chain escape mechanism and see the deviation of experimental results from the purereptation prediction. By formulating the contour-length fluctuation mechanism on the level of the chain and calculating the corresponding structure factor, we are able to quantitatively describe all experimental results and thus for the first time confirm the idea of contour-length fluctuations on a molecular level in space and time.

NSE spectroscopy measures the dynamic-structure factor

$$
S(q, t)=\sum_{m, n}\left\langle\exp \left\{i q\left[r_{m}(t)-r_{n}(0)\right]\right\}\right\rangle
$$

where $q=(4 \pi / \lambda) \sin (\theta / 2)$ is the momentum transfer during scattering ( $\lambda$ : neutron wave length; $\theta$ : scattering angle), $r_{m}(t)$ and $r_{n}(0)$ are the monomer position vectors of monomer $m$ at time $t$ and monomer $n$ at time 0 , the brackets denote the thermal average, and the summation is taken over all monomers of one chain.

Within the framework of the reptation model the two processes identified by de Gennes [1], motion of the 
monomer inside the tube (local reptation) and escape of monomers from the tube (reptation), result in the expression for $S(Q, t)$ :

$$
\frac{S(q, t)}{S(q)}=\left[1-\exp \left(-\frac{q^{2} d^{2}}{36}\right)\right] S^{\mathrm{loc}}+\exp \left(-\frac{q^{2} d^{2}}{36}\right) S^{\mathrm{esc}},
$$

where $S^{\text {loc }}(q, t)$ and $S^{\text {esc }}(q, t)$ are contributions from local motion and escape from the tube, and $d$ is a tube diameter. The local contribution was calculated as

$$
S^{\mathrm{loc}}(q, t)=\exp \left(\frac{t}{\tau_{0}}\right) \operatorname{erfc}\left(\sqrt{t / \tau_{0}}\right),
$$

where $\tau_{0}=36 / W b^{4} q^{4}, b$ is the effective segment length, $W=3 k_{B} T / \xi b^{2}$ is the microscopic attempt frequency (Rouse rate), and $\xi$ is a friction coefficient of one segment. The general expression for $S^{\mathrm{esc}}(q, t)$ due to pure reptation out of the tube was given by Doi and Edwards [2]

$$
\begin{aligned}
S^{\mathrm{esc}}(q, t)= & \sum_{p=1}^{\infty} \frac{2 N \mu}{\alpha_{p}^{2}\left(\mu^{2}+\alpha_{p}^{2}+\mu\right)} \\
& \times \sin ^{2}\left(\alpha_{p}\right) \exp \left(-\frac{4 t \alpha_{p}^{2}}{\pi^{2} \tau_{d}}\right),
\end{aligned}
$$

where $\mu=q^{2} N b^{2} / 12$ and $\alpha_{p}$ are the solutions of equation $\alpha_{p} \tan \left(\alpha_{p}\right)=\mu, \tau_{d}$ is the reptation time, and $N$ is the number of segments. For times shorter than $\tau_{R}=$ relaxation time of an unconfined chain, it was recently shown that the effect of reptation on escaping from the tube is negligible in comparison to tube length fluctuations [7]. This time range corresponds to the times that are accessed in our experiment. For the shortest chain $M_{w}=12.4 \mathrm{~kg} / \mathrm{mol}$ we have $\tau_{R}=186 \mathrm{~ns}$ which is more $N^{2} / \pi^{2} W$, the so-called Rouse time, which is the longest

or less the maximum time reached by NSE. In this regime the fraction of monomers released from the tube due to contour-length fluctuations has a very simple form,

$$
\Psi(t)=\frac{1.5}{Z}\left(\frac{t}{\tau_{e}}\right)^{1 / 4},
$$

where $\tau_{e}=\tau_{R} / Z^{2}$ and $Z=N / N_{e}$ is a number of entanglements per chain. It is straightforward to show that the scaling with $t$ and $Z$ in Eq. (5) emerges exactly from the properties of a linear Rouse chain in a tube. In fact all approaches to contour-length fluctuations so far $[2,8]$ predict for $t<\tau_{R}$ a simple expression for $\Psi(t)$ such as Eq. (5) but with different prefactors $(\approx 1.2$ by Doi and 0.67 by des Cloiseaux), the discrepancy is explained by different mathematical approximations used. We did a series of carefully designed 1D stochastic simulations which solve the first passage problem for the single Rouse chain in a tube, taking the limit of time step tending to zero and the number of monomers going to infinity. These simulations confirmed Eq. (5) and gave a result for the prefactor of $1.5 \pm 0.02$ [7].

To incorporate this result in the structure factor calculations, we used the approximate approach of Clarke and McLeish [9]. We assume that after time $t$ all monomers between 0 and $s(t)$ and between $1-s(t)$ and 1 have escaped from the tube, where $s(t)=\Psi(t) / 2$.

This statement contains two approximations: first, that the fraction of the chain escaped from the tube is the same from each end of the chain, and, second, it ignores the distribution of $s(t)$, replacing it by the single average value. If we make these two assumptions the rest of the calculation is straightforward.

We first use the fact that $r(s, t)$ is a Gaussian variable and therefore

$$
\left\langle\exp \left\{i q\left[r(s, t)-r\left(s^{\prime}, 0\right)\right]\right\}\right\rangle=\exp \left(-\frac{1}{2} \sum_{\alpha=x, y, z} q_{\alpha}^{2}\left\langle\left[r_{\alpha}(s, t)-r_{\alpha}\left(s^{\prime}, 0\right)\right]^{2}\right\rangle\right) .
$$

And then we note that

$$
\left\langle\left[r_{\alpha}(s, t)-r_{\alpha}\left(s^{\prime}, 0\right)\right]^{2}\right\rangle=\frac{d L}{3} \begin{cases}\left|s-s^{\prime}\right| & s(t)<s<1-s(t) \text { or } s(t)<s^{\prime}<1-s(t) \\ \left|2 s(t)-s-s^{\prime}\right| & s<s(t) \text { and } s^{\prime}<s(t) \\ \left|2-s-s^{\prime}-2 s(t)\right| & s>1-s(t) \text { and } s^{\prime}>1-s(t),\end{cases}
$$

where $L=Z d$ is the contour length. The summation in Eq. (1) is then replaced by integration as

$S^{\mathrm{esc}}(q, t)=\int_{0}^{1} d s \int_{0}^{1} d s^{\prime} \exp \left(-2 \mu\left|s-s^{\prime}\right|\right)+2 \int_{0}^{s(t)} d s \int_{0}^{s(t)} d s^{\prime}\left(\exp \left\{-2 \mu\left[2 s(t)-s^{\prime}-s^{\prime}\right]\right\}-\exp \left(-2 \mu s\left|s-s^{\prime}\right|\right)\right)$.

The integrals can be easily evaluated and we obtain

$$
S^{\mathrm{esc}}(q, t)=\frac{N}{2 \mu^{2}}\left[2 \mu+e^{-2 \mu}+2-4 \mu s(t)-4 e^{-2 \mu s(t)}+e^{-4 \mu s(t)}\right] .
$$

A systematic investigation of the molecular weightdependent single-chain dynamic-structure factor from PE melts was performed on samples with altogether six different molecular weights. The PE samples were obtained from parent 1,4-polybutadiene synthesized by anionic polymerization [10]. PE was obtained by subsequent hydrogenation (or deuteration). Each sample contained 10\% protonated PE in a matrix of deuterated PE of similar molecular weight. 
The experiments were performed at the IN15 spectrometer at Institute Laue Langevin in Grenoble covering a range of momentum transfers $0.03 \AA^{-1} \leq q \leq 0.115 \AA^{-1}$ at $T=509 \mathrm{~K}$. The wavelengths of the incoming neutrons were $\lambda=8$ and $15 \AA$ spanning a time range $0.3 \mathrm{~ns} \leq t \leq$ $170 \mathrm{~ns}$. The experimental results were corrected for background and resolution. Figure 1 displays the obtained spectra for different molecular weights and $q$ values.

We first qualitatively discuss the data and compare the results at $q=0.115 \AA^{-1}$. For the highest molecular weights at $M_{w}=190 \mathrm{~kg} / \mathrm{mol}$ and $M_{w}=36 \mathrm{~kg} / \mathrm{mol}$ the spectra are characterized by an initial fast decay reflecting the unconstrained dynamics at early times and very pronounced plateaus of $S(q, t)$ at later times, signifying the tube constraints. The plateau values for the two samples are practically identical. Inspecting the results for smaller $M_{w}$, we realize that (i) the dynamic-structure factor decays to lower values $\left(0.5\right.$ at $M_{w}=190 \mathrm{~kg} / \mathrm{mol}$, 0.4 at $M_{w}=24.7 \mathrm{~kg} / \mathrm{mol}$, and nearly 0.2 at $M_{w}=$ $12.4 \mathrm{~kg} / \mathrm{mol}$ ); (ii) furthermore the long time plateaus start to slope more and more the smaller $M_{w}$ becomes, with $M_{w}=12.4 \mathrm{~kg} / \mathrm{mol}$ nearly losing the two-relaxation-step character of $S(q, t)$. Obviously the chain is disentangling from the tube and constraints are successively removed.

The data were analyzed using both the pure-reptation model of Eqs. (2) and (4), neglecting contour-length fluctuations, and Eqs. (2) and (9), where the contour-length fluctuations are considered. The fits were performed by fixing the elementary Rouse rate $W=411 \mathrm{~ns}^{-1}$, taken from earlier measurements on PE at short times [5], and by varying the tube diameter $d$ as the only parameter.

First the data were analyzed in terms of the purereptation model. For the four higher $M_{w}$, Eqs. (2) and (4) yield a good fit of the data, while for the two lower $M_{w}$ the fit is still acceptable. This is demonstrated in Fig. 2, where the solid lines show the result for the lowest $\left(M_{w}=\right.$ $12.4 \mathrm{~kg} / \mathrm{mol})$ and the highest $\left(M_{w}=190 \mathrm{~kg} / \mathrm{mol}\right)$ molecular weight. For $M_{w}=12.4 \mathrm{~g} / \mathrm{mol}$ and $q=0.115 \AA^{-1}$ the shape of the reptation curve shows significant deviations from the experimental data. The constraint reduction is reflected in the pure-reptation picture by an apparent tube diameter which increases with decreasing chain length [see Fig. $2, d\left(M_{w}=12.4\right)=60 \AA, d\left(M_{w}=190\right)=$ $48.7 \AA$ A. The dotted lines in the lower part of Fig. 2 result from the assumption of a constant $d=48.7 \AA$ inserted in Eqs. (2) and (4); it is obvious that this significantly underestimates the amount of relaxation.

In an alternative approach we took into account the contour-length fluctuations and fitted the data with Eqs. (2) and (9). The lines in Fig. 1 display the result. A group of three common $q$ values for all molecular weights is displayed by thick solid lines to facilitate an easy comparison of the molecular weight dependence of the curves. Additional $q$ values only available for some $M_{w}$ 's are represented by dotted lines. The open squares in Fig. 3 show the resulting tube diameters. Aside from some small fluctuations they now stay constant, independent of $M_{w}$. We

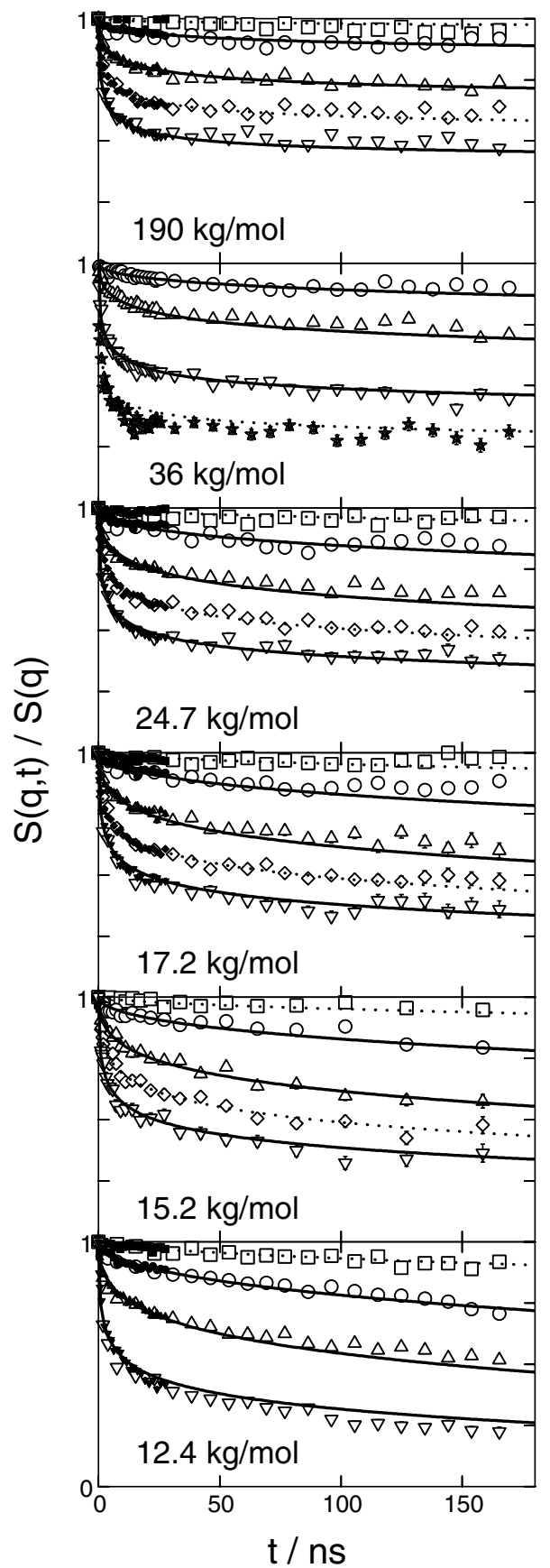

FIG. 1. Neutron-spin-echo spectra from polyethylene melts of various molecular weights. The $q$ values correspond to squares, $q=0.03 \AA^{-1}$; circles, $q=0.05 \AA^{-1}$; triangles (up), $q=0.077 \AA^{-1}$; diamonds, $q=0.096 \AA^{-1}$; triangles (down), $q=0.115 \AA^{-1}$; crosses, $q=0.15 \AA^{-1}$. Filled symbols refer to a wavelength of the incoming neutrons $\lambda=8 \AA$ and open symbols refer to $\lambda=15 \AA$. For lines, see explanation in text.

further note that in particular for smaller $M_{w}$ the weighted error between fit and data is significantly smaller for this second approach.

If we compare the experimental spectra with the model prediction [Eqs. (2) and (9)], we generally find good agreement. The gradually increasing decay of $S(q, t)$ with decreasing $M_{w}$ is depicted very well both with respect to 


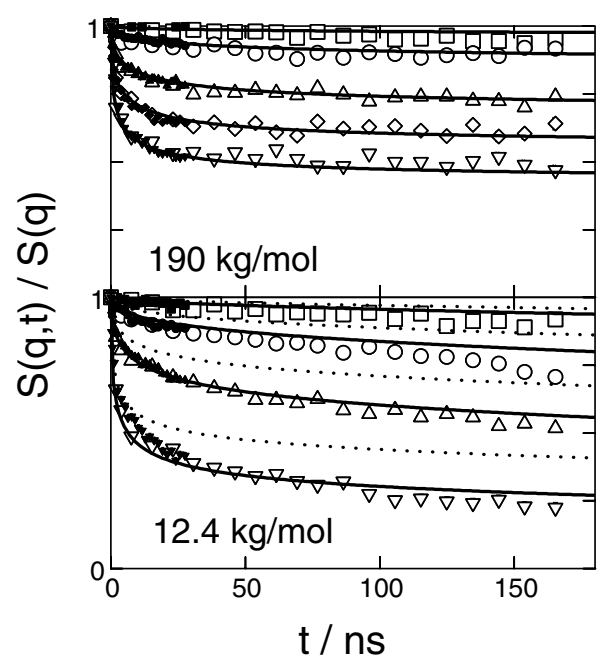

FIG. 2. Data as in Fig. 1. Solid lines represent a fit with purereptation model leading to $d\left(M_{w}=190 \mathrm{~kg} / \mathrm{mol}\right)=48.7 \AA$ and $d\left(M_{w}=12.4 \mathrm{~kg} / \mathrm{mol}\right)=60 \AA$. For the dotted lines the tube diameter was fixed to $48.7 \AA$.

the magnitude of the effect as well as to the shape of $S(q, t)$. The largest disagreement is seen in the $M_{w}=$ $17.2 \mathrm{~kg} / \mathrm{mol}$ data, where at lower $q$ the data appear to be somewhat irregular. At $q=0.05 \AA^{-1}, S(q, t)$ tends to increase at longer times, possibly reflecting some systematic problems with measurement.

We now consider the tube diameters resulting from the two approaches (Fig. 3). At the highest molecular weight, contour-length fluctuations are insignificant and both lines of fitting yield the same $d$. At $M_{w}=36 \mathrm{~kg} / \mathrm{mol}$ a slight difference appears which increases strongly with decreasing length. At $M_{w}=12.4 \mathrm{~kg} / \mathrm{mol}$ the difference in the fitted tube diameters between both approaches rises to nearly $50 \%$, emphasizing the strong effect of the contour-length fluctuation in loosening the grip of the entanglements on a given chain.

Thus, the comparison between the experimental chainlength-dependent dynamic-structure factor and theoretical predictions clearly shows that, in the time regime $t \leq \tau_{R}$, contour-length fluctuations are the leading mechanisms that limit the chain confinement inherent to the reptation picture. Without any further assumption or fitting parameter - the tube diameter $d$ stays constant with $M_{w}$ - it is possible to describe the full $M_{w}$ dependence of $S(q, t)$ in terms of local reptation and the contour-length fluctuation mechanism. Even for chain lengths corresponding to only 6-7 entanglements the tube diameter appears to be a well-defined quantity assuming the same value as for asymptotically long chains. The confinement is lifted from

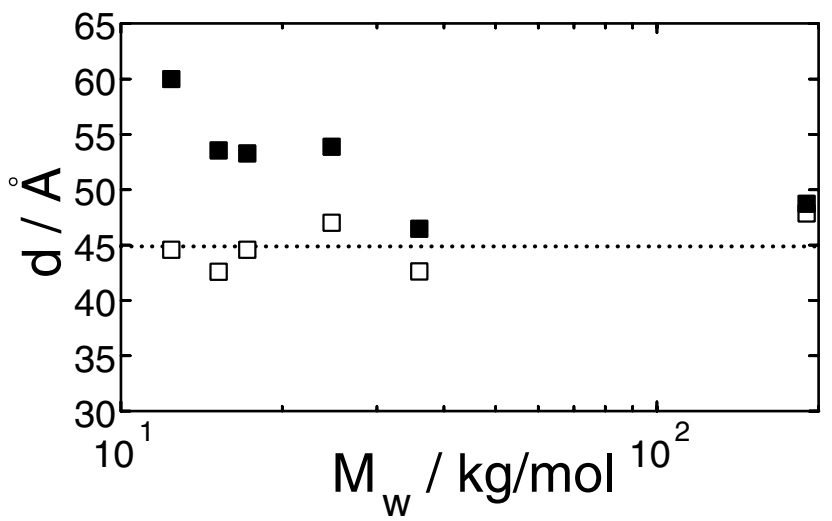

FIG. 3. Resulting tube diameters from the model fits with pure reptation (filled squares) and reptation and contour-length fluctuations (open squares) as a function of molecular weight. The dotted line is a guide for the eye.

the chain ends inwards, while the chain center remains confined in the original tube.

In conclusion, our study of the chain-length-dependent dynamic-structure factor in PE melts provides strong support to a theory of contour-length fluctuations as the confinement limiting mechanism on a molecular scale. There is no significant dependence of the tube diameter on the chain length. We also note that generalized Rouse models, such as that conceived by Hess [11], do not describe the data. Further experiments on chains with labels in the chain center will further substantiate the picture. Future theoretical work will include an analysis of preaveraging approximations used to derive Eq. (9) as well as calculations for partially labeled chains.

[1] P. G. DeGennes, J. Physique 42, 735 (1981).

[2] M. Doi and S. F. Edwards, The Theory of Polymer Dynamics (Clarendon Press, Oxford, 1988).

[3] M. Doi, J. Polym. Sci. Polym. Lett. Ed. 19, 265 (1981).

[4] D. Richter, R. Butera, L. J. Fetters, J. S. Huang, B. Farago, and B. Ewen, Macromolecules 25, 6156 (1992).

[5] D. Richter, B. Farago, R. Butera, L. J. Fetters, J. S. Huang, and B. Ewen, Macromolecules 26, 795 (1993).

[6] P. Schleger, B. Farago, C. Lartigue, A. Kollmar, and D. Richter, Phys. Rev. Lett. 81, 124 (1998).

[7] A. E. Likhtman and T. C. B. McLeish (to be published).

[8] J. des Cloiseaux, Macromolecules 23, 4678 (1990).

[9] N. Clarke and T. C. B. McLeish, Macromolecules 26, 5264 (1993).

[10] M. Morton and L. J. Fetters, Rubber Chem. Technol. 48, 359 (1975).

[11] W. Hess, Macromolecules 21, 2620 (1988). 